\title{
Hyperinnervation Produces Inhibitory Interactions between Two Taste Nerves $^{a}$
}

\author{
D. R. RIDDLE, B. OAKLEY, S. E. HUGHES, C. L. \\ DESIBOUR, AND C. R. BELCZYNSKI \\ Department of Biology \\ University of Michigan \\ Ann Arbor, Michigan 48109
}

\begin{abstract}
Several researchers have presented evidence that suggests that the processing of gustatory information at the level of the taste bud can involve interactions among taste fibers, for example, Bernard ' and Miller. ${ }^{2}$ We report here that two chorda tympani nerves in the same peripheral field may interact to inhibit gustatory impulse traffic to the brain.
\end{abstract}

\section{METHODS}

The proximal portion of the right chorda-lingual nerve was sutured to the distal stump of the left lingual nerve in $\mathbf{4 4}$ mongolian gerbils. Nine to fifteen months after the initial surgery, a second operation was performed on six gerbils. The native chorda tympani was removed in four gerbils and the foreign chorda tympani was removed in two. In 10 control animals the left lingual nerve and the right chorda-lingual nerve were transected. The latter was reconnected with an 11-0 suture to allow reinnervation of the right side of the tongue. Five to twenty months after the initial surgery, the chorda tympani nerves were exposed in the left and right middle ears and impulse activity simultaneously recorded from the two nerves while stimulating the tongue with a variety of taste solutions. It was also possible to electrically stimulate one chorda tympani while simultaneously recording from the other. Each animal was sacrificed at the end of the recording session. The tongue was removed and processed for light microscopy. Fungiform taste buds were counted and their positions on the tongue noted. A subset of taste buds was analyzed morphometrically. Counts of taste cells were made from plastic sections of fungiform taste buds from three experimental and four normal animals.

\footnotetext{
${ }^{a}$ Supported in part by United States Public Health Service Grant NS-07072.
} 


\section{RESULTS AND DISCUSSION}

Punctate taste stimulation of the tip of the left side of the tongue in experimental animals elicited responses in both chorda tympani nerves. Stimulation of the right side was effective only after the taste solutions spread. Both nerves must have innervated the left side of the tongue. Histological analysis showed a near absence of fungiform taste buds on the right side of these tongues, with the exception of an occasional taste bud near the tip or along the midline. The eventual removal of the native or foreign chorda tympani showed that the native nerve alone supported all of the fungiform taste buds on the left side of the tongue, and that the foreign nerve would maintain about half of those taste buds. It appears then, that at least half of the fungiform taste buds on the left side of the tongue were dually innervated. Both the native and foreign chorda tympani nerves responded vigorously to taste stimulation and the concentration response functions were similar. However, simultaneous recording from the nerves revealed a number of response characteristics indicative of inhibition that were most profound in the foreign nerve. In $75 \%$ of the animals, responses of the foreign nerve to a variety of taste stimuli were both slower to rise and slower to return to baseline compared to responses in the native nerve. A 5-10fold increase in the time required to reach $90 \%$ of peak height was not uncommon. Inhibition was observed in approximately three-fourths of the animals and was always much more pronounced in the foreign nerve. The vigor of inhibitory interactions varied unsystematically among animals and the taste solutions used as stimuli. In some instances a brief transient response was followed by a slow, delayed increase in activity. In other instances a transient, but unsustained, response was present. In the most dramatic cases, taste stimulation failed to elicit even a transient response and actually depressed spontaneous activity. With more concentrated taste solutions, this initial depression of spontaneous activity was slowly overcome and a neural discharge emerged. We observed none of these inhibitory interactions in control or normal animals.

Typically, in the same experimental animals whose foreign chorda tympani nerve displayed the inhibitory responses described above, we found that electrical stimulation of the native nerve depressed subsequent taste responses in the foreign nerve. Responses to both sucrose and $\mathrm{NaCl}$ were often suppressed by at least $35 \%$. The effect was usually maintained for several minutes and, like the inhibition described above, was still observed after the native nerve was disconnected from the brain. It seems unlikely that the inhibition resulted from general autonomic effects, such as a change in blood flow through the papillae, since no change was seen in the response of the native nerve following electrical stimulation of either the native or foreign nerve. Since both nerves innervated many of the same papillae, one would expect them to respond similarly to autonomic influences. We observed no inhibitory effects of electrical stimulation in normal or control animals.

The anatomical substrate underlying these inhibitory interactions remains to be determined. Nor is it clear that inhibition by electrical stimulation operates through the same mechanisms as the inhibition observed during taste stimulation. Light microscopy revealed no anatomical changes that might account for the unusual physiological effects. Hyperinnervation failed to increase the number of taste buds, the size of taste buds, or the number of cells within taste buds. This is somewhat surprising given the clear trophic influence of taste neurons upon taste buds, and suggests that it is the character of the gustatory epithelium, not the density of innervation, that 
controls the number and size of fungiform taste buds. Electron microscopic studies now being conducted (Kinnamon et al., unpublished) may reveal ultrastructural changes that could be responsible for the pronounced inhibitory interactions observed.

\section{REFERENCES}

1. Bernard, R. A. 1972. Antidromic inhibition: A new model of taste receptor function. In Olfaction and Taste IV. D. Schneider, Ed.: 301-307. Wissenschaftliche Verlagsgesellschaft MBH. Stuttgart, Germany.

2. Miller, I. J. 1975. Mechanisms of lateral interactions in rat fungiform taste receptors. In Olfaction and Taste V. D. A. Denton \& J. P. Coghlan, Eds.: 217-221. Academic Press, Inc. New York. 